\title{
Electric Currents and Potentials Resulting From the Flow of Charged Liquid Hydrocarbons Through Short Pipes
}

\author{
M. R. Shafer, D. W. Baker, and K. R. Benson
}

(June 21, 1965)

\begin{abstract}
The electrical currents and potentials produced in pipes of intermediate and very high resistivities, by the flow of a charged liquid hydrocarbon have been investigated. The maximum pipe currents to the ground were in the range 1 to 6 microamperes. Depending upon the electrical resistance of the pipes, these currents produced potentials ranging from essentially zero to values in excess of 30,000 volts which were sufficiently severe to cause electrical breakdown and ares within some of the pipes under investigation. It is concluded that hazardous pipe potentials, resulting from static electricity, can be eliminated in practical applications if the electrical resistance from each and any portion of the interior surface of the pipe to the ground does not exceed about $10^{7} \mathrm{ohms}$.
\end{abstract}

Key Words: Breakdown, charged liquid hydrocarbons, charging tendency, charge separation, current, filters, inner liner, petroleum liquids, potential, relaxation, resistance, static electricity, tetrafluoroethylene tubing.

\section{Introduction}

The petroleum industry has long been concerned with the problem of static electricity resulting from the flow of relatively nonconducting liquid hydrocarbons through pipes, filters, and other components. Considerable literature is available which considers methods by which such charges are produced and the hazards arising from the introduction of electrically charged petroleum liquids into receiving tanks, as frequently occurs during refinery and fueling operations.

Problems related to the electrical currents and potentials produced in pipes and hoses through which charged petroleum liquids flow have not been investigated thoroughly. Carruthers and Marsh [1] ${ }^{1}$ and Carruthers and Wigley [2] considered this problem recently and developed equations relating the currents and potentials produced with the electrical resistance of the pipe, the electrical characteristics of the fuel and its velocity. Experimental checks were performed using pipes of essentially infinite and zero electrical resistance. Although these conditions were well suited for investigations of charge relaxation, neither condition provided experimental verification of the potentials produced in pipes having large, but not infinite, values of electrical resistance.

Under the sponsorship of the Department of the Navy, Bureau of Naval Weapons, the National Bureau of Standards has conducted an investigation to determine the desirable electrical resistance charac-

${ }^{1}$ Figures in brackets indicate the literature references on page 316. eristics of hoses used to interconnect the various fuel handling components of aircraft and other internal combustion engines. It is believed that some of the results of the investigation are of sufficient general interest for presentation here as they provide further experimental verification of the theory and ideas presented in [1] and [2] and their Discussions.

The experimental investigations to be described were concerned primarily with a determination of the magnitude of electrical currents and voltages encountered in pipes of intermediate and very high resistivities through which electrically charged liquid petroleum flows. The pipes had a nominal inside diameter of 0.875 -in. and lengths in the range of 20 to 48 in. Flowrates of 5 to 30 gal per minute were used with fuels having conductivities of 8 to 200 picomho per meter. Pipe currents to the ground encompassed the range $10^{-8}$ to $6 \times 10^{-6} \mathrm{~A}$. Depending upon the electrical resistance of the pipes, these currents produced potentials ranging from essentially zero to values in excess of $30,000 \mathrm{~V}$, which were sufficiently high to cause electrical breakdown and arcs within some of the pipes under test.

\section{Theoretical Considerations}

Consider a pipe of length $L$, as shown in figure 1 , through which a relatively nonconducting liquid is flowing at a mean axial velocity $v$. The liquid entering the section may or may not contain a significant initial charge $q_{i}$ per unit length of pipe which corresponds to a convection current $I_{i}=q_{i} v$ entering the section under consideration. Likewise the liquid leaves the pipe with a charge $q_{u}$ per unit length of 


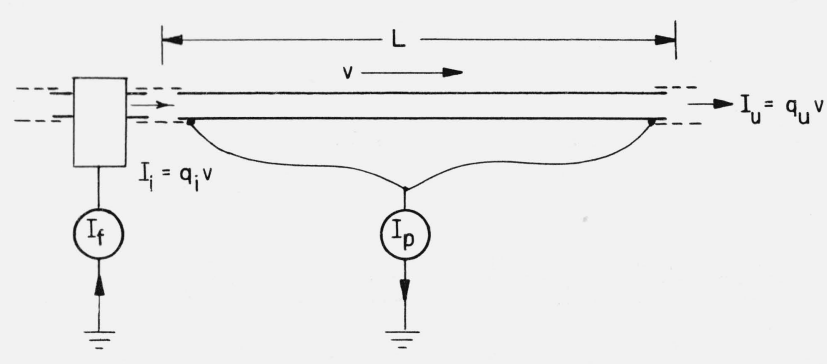

FIGURE 1. Electric currents resulting from the flow of a fluid.

pipe causing an exit convection current $I_{u}=q_{u} v$. As discussed in [1], the value of $q_{u}$ and hence $I_{u}$ is dependent upon: the magnitude of $\boldsymbol{I}_{i}$, the resistivity of the fuel, the residence time, $t$, during which the fuel remains within the length of pipe, and the amount of charge separation which occurs at the fuel-pipe interface.

The equilibrium current $I_{p}=I_{i}-I_{u}$, flowing from the pipe to the ground, is a direct measure of the net rate at which charges are deposited on the interior surface of the pipe during passage of the fuel. It is conveniently measured by electrically insulating the pipe section from the remainder of the flow circuit and from the ground, and electrically connecting each end of this section to the ground through a suitable ammeter.

Convenient instrumentation for the direct measure of $I_{i}$ is not available. However, when a chargeproducing component such as a microfilter is placed immediately upstream of the test section, the current $I_{f}$ between the filter and the ground is approximately equal to $I_{i}$. This filter current is conveniently measured by an ammeter if the filter is electrically insulated from the remainder of the flow circuit. Thus, $I_{f}=I_{i}$ provided the fuel entering the filter is essentially uncharged.

The relation between $I_{p}$ and $I_{i}$, as developed in the appendix, is

$$
I_{p}=\left(I_{i}-I_{0}\right)\left(1-e^{-\lambda}\right)
$$

where $I_{0}$ is the convection current that would emerge with the liquid from an infinitely long pipe under the same conditions. If $I_{i}$ is caused by a filter placed immediately upstream from the test section and is large compared to $I_{0}$, then the relation

$$
I_{p} \approx I_{f}\left(1-e^{-\lambda}\right)
$$

should be correct for the more severe charging conditions.

The term $\lambda$ is the ratio of resistance time, $L / v$, of the liquid in the pipe to relaxation time, $\tau$, of the liquid. Relaxation time is the time required for the charge existing in the stationary fuel to be reduced 63 percent in magnitude by electrostatic forces.

Considering a pipe grounded at each end, an approximate relation for the maximum potential devel- oped on the pipe wall, as a result of current $I_{p}$, is

$$
V_{\max }=0.1 R_{T} I_{p},
$$

where $R_{T}$ is the end-to-end resistance. Commencing with an expression from [1] for the voltage developed on the pipe wall at any distance from the inlet, this relation has been developed in the appendix subject to the following assumptions:

(a) The ratio $\lambda$ has numerical value less than 6 .

(b) The electrical resistance $R_{T}$ of the pipe is small compared to that of the liquid contained within the pipe.

The constant 0.1 of eq (3) varies through the approximate range 0.08 to 0.13 for values of $\lambda<6$. Values of $\lambda>6$ are encountered only with long pipes, fuel of very high conductivities, or very low fuel velocities for which conditions nearly all of the separation of charges, $q_{i}$, occurs near the entrance of the pipe. Values of $\lambda<6$ are those normally encountered during the flow of liquid petroleum products, and a constant factor of 0.1 can be selected for this range of $\lambda$ with little sacrifice in accuracy.

The assumption that pipe resistance $R_{T}$ is small compared to that of the liquid within the pipe is valid for pipes having resistances no greater than $10^{10} \Omega$ per linear inch of length even when fuels having conductivities as large as $200 \mathrm{pmho} / \mathrm{m}$ are involved. Pipes having higher electrical resistances are not considered desirable because of the possibility of electrical discharges resulting from the extremely high voltages produced. Thus, it is believed the assumptions involved in deriving the approximate relation expressed in eq (3) are valid as far as practical applications are concerned.

It is of interest to note that eq (3) is essentially identical to the relation $V_{\max }=0.106\left(I_{i}-I_{0}\right) R_{T}$ which was presented by Dr. Klinkenberg in the Discussion of [2].

Data were obtained during these experiments which verifies eqs (2) and (3) using pipe specimens having $R_{T}$ values in the range zero to $10^{10} \Omega$. Also, the influence of the electrical resistance of the pipe on the current $I_{p}$ was investigated; and the electrical breakdown phenomena which occur when large values of $I_{p}$ are encountered in pipes having extremely high values of $\boldsymbol{R}_{T}$ were observed.

\section{Experimental Apparatus}

The flow circuit, shown in figure 2, included a supply tank containing 50 gal of naptha and a centrifugal pump of $30 \mathrm{gpm}$ capacity. The flow then passed through a suitable flowmeter into the microfilter used for the generation of the initial charge $q_{i}$. A by-pass valve was installed to provide a method of directing the flow through or around the filter as desired. The pipe sample under test was connected immediately downstream from the filter and the fuel then returned through a flow control valve to either the supply or receiver tank as desired. Each end of the pipe sample, as well as the entrance connection to the 
filter, was electrically insulated from the flow system by short lengths of nonconductive tetrafluoroethylene (TFE) tubing. Short adapters, of metal tubing, were used as connectors between the ends of the test pipe and the insulating tubing. They were inserted into the ends of the test pipe to a depth of about one inch, thus forming internal electrodes. These two electrodes were electrically connected to each other and to the ground through an ammeter for the measurement of $I_{p}$. Likewise, the filter was connected to the ground through another ammeter for the measurement of $I_{f}$. All other portions of the flow system were constructed from conductive materials and grounded as in normal service.

The liquid used throughout the tests was a waterwhite, hydrocarbon naptha designated as MIL-F7024 A, Type II. This naptha has a $60 / 60^{\circ} \mathrm{F}$ specific gravity of 0.771 , a kinematic viscosity at $80^{\circ} \mathrm{F}$ of about 1.2 centistokes, an initial distillation point above $300{ }^{\circ} \mathrm{F}$, and a flash point above $100^{\circ} \mathrm{F}$. The electri cal conductivity of the naptha as received, in 55-gal drums, at our laboratory is about $1 \mathrm{pmho} / \mathrm{m}$. Contaminants were added to obtain the appreciable filter charging effects required.

Two different filters were used. Each had an external metal body containing a phenolic resin impregnated paper element. One filter had a $25-\mu \mathrm{m}$ size element of a pleated design containing a total of 35 pleats 1 -in. deep by 4.5 -in. long giving a total filter surface area of about $300 \mathrm{in.}^{2}$ The nominal flow rating of this element was $25 \mathrm{gpm}$ with a fuel of 1 centistroke viscosity. The second filter had a $10-\mu \mathrm{m}$ size element of a stacked-paper washer design having a length of 4 in., an outside diameter of 1.5 in., giving a total filter surface area of about $18 \mathrm{in.}^{2}$ This element had a nominal flow rating of $5 \mathrm{gpm}$ with a fuel of 1 centistoke viscosity. Both of these filters and their elements were identical in size and design to those commonly used in fuel systems of some internal combustion engines.

The ammeters used for the measurement of $I_{f}$ and $I_{p}$ were multipurpose electrometers having a range $10^{-13}$ to $3 \mathrm{~A}$ full scale. The voltage drop of these instruments never exceeded $1 \mathrm{~V}$ during the measurement of current.

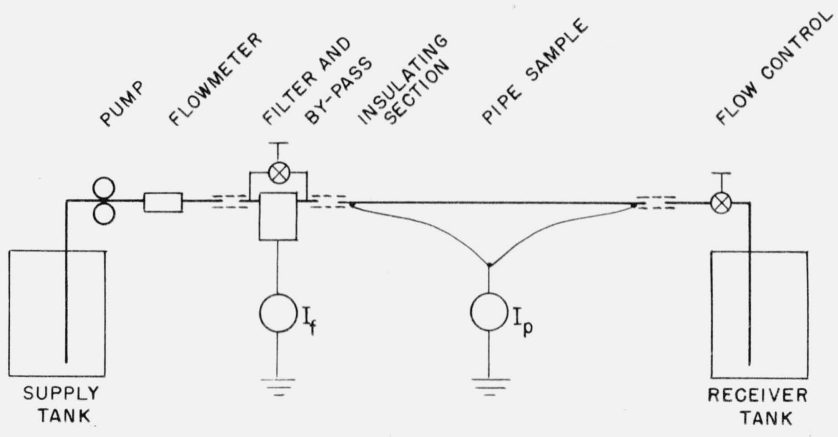

FigURE 2. Diagram of the flow circuit.
Pipe potentials of less than $100 \mathrm{~V}$ were measured with a multipurpose electrometer having a resistance to ground of the order of $10^{14} \Omega$. Voltages in the range 100 to $10,000 \mathrm{~V}$ were measured with electrostatic voltmeters having resistances to ground of the order of $10^{16} \Omega$. No attempts were made to measure potentials exceeding $10,000 \mathrm{~V}$ as corona effects with the resulting breakdown of resistance were always encountered in this region.

Pin-type probes were constructed for the measurement of the potentials developed on the inner surfaces of the pipe walls. As will be discussed in detail, the test specimens were tetrafluoroethylene (TFE) tubing containing an inner liner of intermediate resistivity and having a total wall thickness of 0.040 -in. The probes contained pin points about 0.06-in. long and were inserted through the tubing from the outside, thus puncturing and making electrical contact with the inside conductive liner of the pipe. Two pins pierced the wall at each probe location.

Fuel conductivities were determined by a conductivity cell constructed in accordance with figure 8 of reference [3]. The actual plate separation was 7.9 $\mathrm{mm}$. A constant test potential of $150 \mathrm{~V}$ dc was used resulting in a test potential gradient of $19 \mathrm{~V} / \mathrm{mm}$. Significant polarization was obtained during the determination of fuel conductivities as evidenced by a considerable and continuous decrease in current with time under the condition of a constant applied voltage. For comparative purposes, the current used to compute fuel conductivity was that occurring $30 \mathrm{sec}$ after applying the test voltage to the cell. Experience with this and other methods of conductivity measurement indicates that the determination of fuel conductivity is not an exact art.

\section{Test Specimens}

The pipe samples used during the investigation had nominal inside diameters of 0.875 -in. and their lengths were in the range 20 to $48 \mathrm{in}$. One sample was a 22 -in. length of stainless steel tubing which for purposes of this investigation is considered to have zero electrical resistance. All of the other samples were formed from the thermoplastic tetrafluoroethylene (TFE) which in a pure state has a volume resistivity $>10^{18} \Omega-\mathrm{cm}$. Each sample of TFE pipe had a wall thickness of about 0.040 -in. The dielectric strength of the pure TFE was such that electrical breakdown would not occur when a potential of $30,000 \mathrm{~V}$ de was applied across the wall.

Experimental samples of intermediate resistivity TFE tube were produced for use in this investigation by members of the aircraft hose industry. These differed from conventional, pure TFE in that each contained an inner layer to which small amounts of carbon had been added. This resulted in an inner liner of moderate conductivity, about 0.010 -in. thick, extending the full length of the pipe and attached to and surrounded by an outer wall of nonconductive TFE about 0.030-in. thick. Thus, electrical conduction was in the longitudinal direction only. 


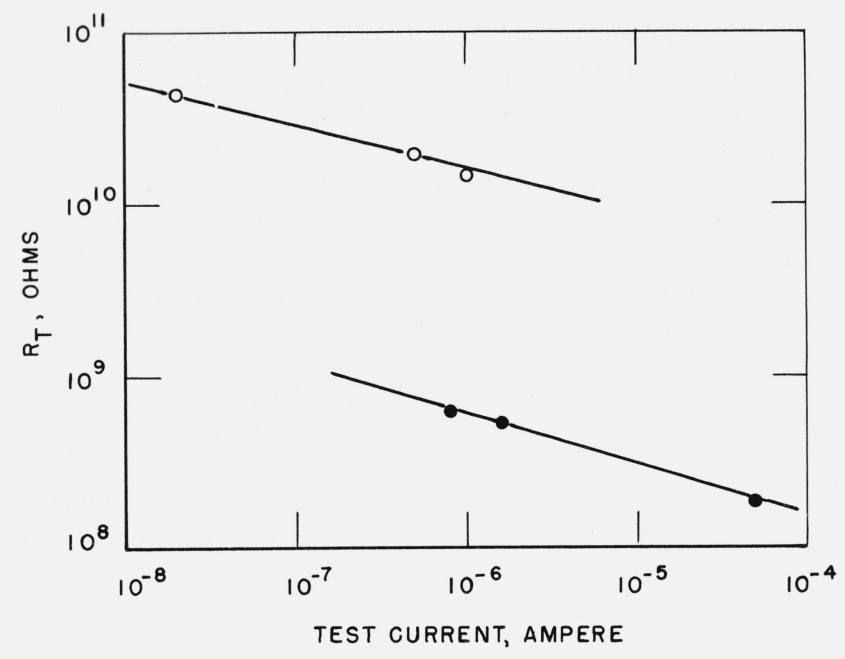

FIGURE 3. End-to-end resistance of two samples of TFE pipe with carbon inner liners showing the variation in resistance with current.

$$
\text { ○ Sample A, } \quad \text { Sample B }
$$

Pipes made in this manner have a voltage dependent, nonlinear, electrical resistance which tends to decrease appreciably with increased potential gradient. Thus, in performing tests to determine the ohmic resistances of the samples, it was found that current is a nonlinear function of the applied voltage. Typical results of end-to-end resistance tests of two of the pipe samples, 36 in. long, are shown in figure 3 . These resistance values were obtained by applying selected constant test potentials across the pipe sections and measuring the resulting current. Unless stated otherwise, $R_{T}$ values listed in this paper are those obtained with a test current of $1 \mu \mathrm{A}$.

\section{Tests and Results}

\subsection{Fuel Contamination and Filter Charging Tendency}

Initial tests were conducted to investigate the relation between fuel contamination and the charging tendency of paper microfilters. The flow circuit, as shown in figure 2, utilized a two-tank system to enable use of one tank for supply and the other as a receiver. This system is desirable for experiments with fuels of low conductivities as it ensures uncharged fuel entering the pump inlet line. Thus, service operating conditions are duplicated closely. In these tests, a 48-in. length of semiconductive TFE tube $\left(R_{T}=50 \mathrm{M} \Omega\right)$ was used as the pipe sample, and the filter current, $I_{f}$, and pipe current $I_{p}$ to the ground were measured.

The naptha had an initial conductivity of about $1 \mathrm{pmho} / \mathrm{m}$. The two contaminants investigated were asphaltenes and a commercial antistatic compound (ASC). Fuel conductivities in the range 2 to 200 pmho/m were obtained by varying the concentration of these two materials. The asphaltenes were dispersed quickly and evenly throughout the test naptha by first dissolving in isopropylbenzene (Cumene). In order to generate large charging currents, these initial tests were performed with the 5 -gpm stacked paper filter at a flowrate of $8 \mathrm{gpm}$ through the filter. This corresponds to an average velocity of $4 \mathrm{ft} / \mathrm{sec}$ across the section of the $0.875 \mathrm{i}$. d. TFE pipe specimen.

The results of the stacked-paper filter charging tendency tests are shown in figure 4, a plot of filter current versus the fuel conductivity. Contamination with asphaltenes gave very significant filter charging currents with fuel conductivities above $10 \mathrm{pmho} / \mathrm{m}$. The maximum charging tendency obtained by contamination with the antistatic compound (ASC) was only about $1 / 25$ of that obtained with the asphaltenes under identical test conditions. Also, with the ASC contaminant, charging tendency decreased rapidly to a low value as conductivity exceeded $100 \mathrm{pmho} / \mathrm{m}$. One additional point on figure 4 shows the effect of adding a small amount of the ASC to the asphaltenes mixture having an original conductivity of about $200 \mathrm{pmho} / \mathrm{m}$. A considerable decrease in charging tendency was noted with a relatively small increase in fuel conductivity.

The charging tendency of the napthas containing the asphaltenes and ASC contaminants were also studied using the apparatus described on page 50 of reference [3]. Briefly, this provides a method of determining the amount of electric charge produced as a fuel flows by gravity through a steel capillary having an inside diameter of $3 \mathrm{~mm}$ and a length of

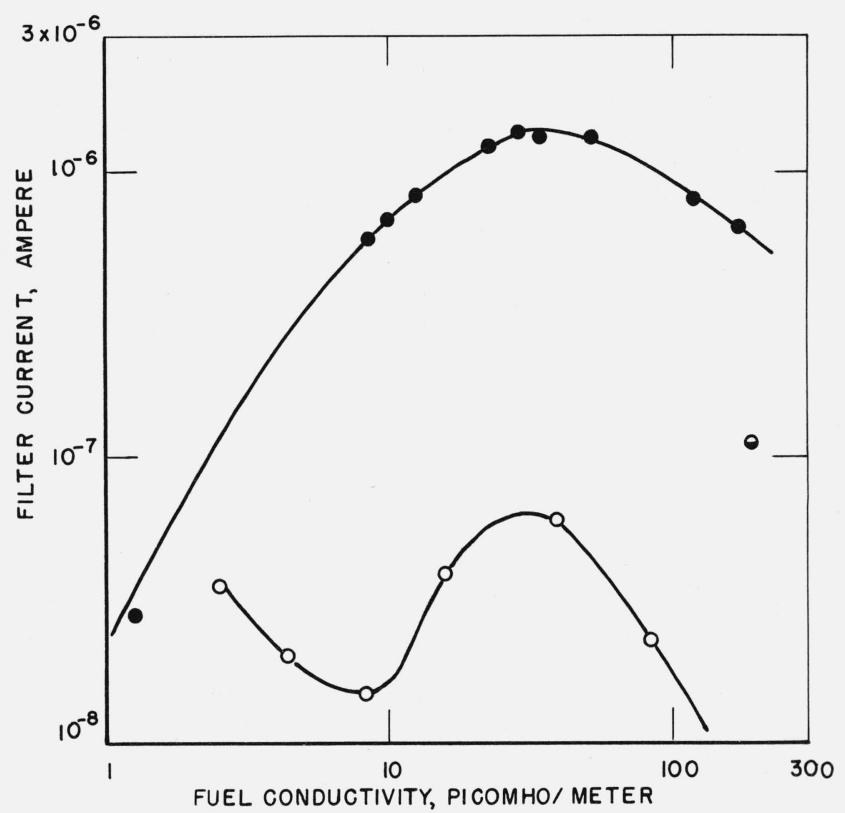

FIGURE 4. Charging tendency of a stacked-paper filter at a constant flowrate of $8 \mathrm{gpm}$ showing the influence of fuel conductivity with two different fuel contaminants.

- Asphaltenes, $\bigcirc$ ASC Antistatic Compound, Asphaltenes plus ASC. 
$0.5 \mathrm{~m}$. No relationship was found to exist between charging tendencies obtained with the paper filter as compared to those obtained using the steel capillary. In fact, the ASC contaminant gave approximately the same charging tendencies in the steel capillary as was obtained with asphaltene contamination.

Also in tests of other fuels having conductivities of 84 and 165 pmho/m caused by unknown contaminants, relatively insignificant charging tendencies were indicated by tests with the steel capillary while extremely large charging currents were obtained when the same fuels flowed through paper microfilters.

Thus, the investigation of the relation between contamination, fuel conductivity, and charging tendency showed that the charging tendency of a given liquid hydrocarbon is not related to fuel conductivity alone. Rather, as might be expected, it is dependent upon both the material and the amount of the contaminant in the liquid and upon the wall material at the liquid-wall interface. The investigation also showed that maximum charging tendency in microfilters will likely occur when fuel conductivity exceeds $10 \mathrm{pmho} / \mathrm{m}$.

\subsection{Preliminary Tests With Various Pipe Samples}

Preliminary experiments were conducted with various pipe samples to determine: (a) the magnitude of pipe currents to be expected from pipes installed immediately downstream from the microfilters; (b) the influence of flowrate upon these pipe currents; and (c) whether the electrical conductivity of the pipe had a significant influence upon these currents. Fuel having a conductivity of $165 \mathrm{pmho} / \mathrm{m}$ was used throughout the preliminary tests. Pipe current, $I_{p}$, was measured, but unfortunately, the filter current, $I_{f}$, was not measured during the preliminary tests. However, an effort was made to keep the test conditions the same as the different pipe specimens were investigated. Thus, it can be assumed that the convection currents, $I_{i}$, entering the different test specimens were approximately equal at identical flowrate conditions. Only the pleated-paper filter was used during these tests. The filter by-pass valve in figure 2 was closed; thus all flow passed through the filter.

The pipe specimens selected for the preliminary tests all had a length of 22 in. These included a stainless steel tube and two samples of TFE pipe containing carbon inner liners having $R_{T}$ values of $10^{6}$ and $10^{9} \Omega$. Voltage measurements were not made and the pin-type probes were not applied to the test samples.

The results of the preliminary tests are shown in figure 5 . It is seen that $I_{p}$ increased significantly with flowrate and that the largest values observed were in the range 2 to $6 \mu \mathrm{A}$. Also, the pipe currents were about equal in magnitude for all values of pipe resistance investigated. Thus, it was verified (as might be expected) that pipe resistance has very little influence, if any, upon the rate of relaxation of charges from the charged fuel to the walls of the pipe.

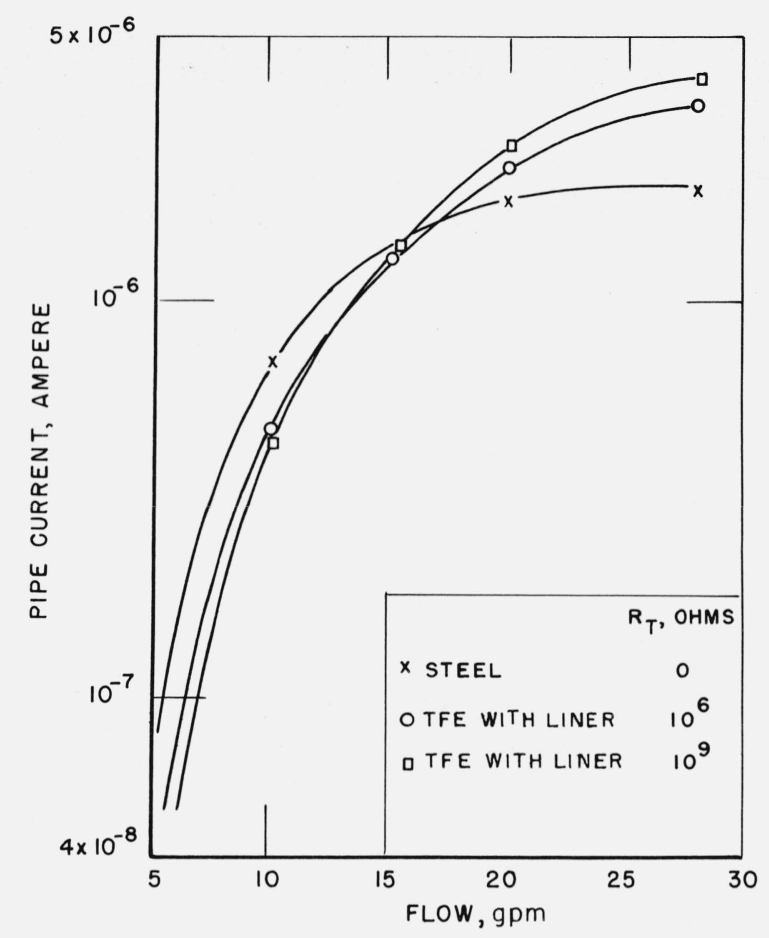

FIGURE 5. Current to the ground from pipes of different resistivities, as influenced by flowrate (each pipe 22-in. long installed downstream from a pleated-paper filter, fuel conductivity 165 pmho/m).

\subsection{Electrical Breakdown in Nonconductive Pipes}

When a nonconductive TFE pipe sample 22-in. long, was inserted in this same flow circuit, electrical sparks and other discharges occurring from the sample were observed. Also pinhole punctures appeared in the walls of the sample through which fuel slowly seeped to the atmosphere. The conditions leading to the electrical breakdown of the pure TFE pipe follow.

An unpunctured, translucent sample of bare TFE pipe 22 -in. long, wall thickness 0.040 -in., was installed downstream from a paper microfilter. The sample was electrically insulated from the remainder of the flow system by short lengths of TFE tubing. Short metal adapters were inserted between each end of the sample and the insulating tubing. These two adapters were electrically connected to each other and to the ground. A probe for measuring electrostatic potential was installed on the outside at the center of the sample. This consisted of a TFE insulator bushing, 0.25-in. wall thickness, having an aluminum foil strip wrapped around the exterior and connected to an electrostatic voltmeter. There was no other grounded metallic object close to the TFE pipe.

With the room darkened so electrical discharges could be seen more readily and using a fuel having a conductivity of about $165 \mathrm{pmho} / \mathrm{m}$, flow through the system was quickly adjusted to $15 \mathrm{gpm}$ at $25 \mathrm{psig}$. 


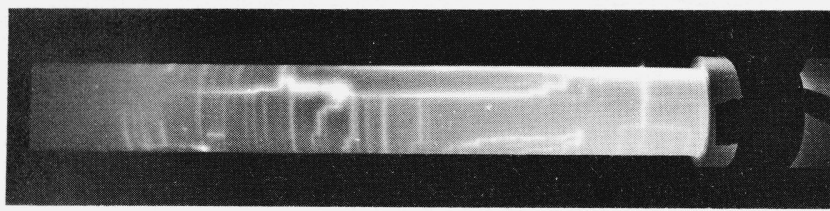

FigURE 6. Electrical discharges in a pure TFE pipe connected downstream from a pleated-paper filter (flowrate $15 \mathrm{gpm}$, fuel conductivity $165 \mathrm{pmho} / \mathrm{m})$.

The electrostatic voltmeter rose rapidly to a maximum of about $8 \mathrm{kV}$ and then oscillated about this value indicating that some electrical discharges were likely occurring. These preliminary discharges were not visible in the darkened room.

After a period ranging from about 1 to $4 \mathrm{~min}$ at this constant flow condition, a very bright electrical discharge took place inside of the pipe. It consisted of bright flashes running lengthwise inside of the tube with numerous lateral flahses branching off as shown in the photograph of figure 6 . After this initial discharge, the voltmeter oscillated about the $5 \mathrm{kV}$ point, and the wall of the test pipe contained numerous pinhole punctures through which fuel slowly seeped.

Light for the photograph of figure 6 was obtained entirely from the electrical discharge which took place within the translucent pipe sample. The actual portion of the pipe shown is the upstream part with the entrance to the left and the TFE insulator bushing of the voltage probe at the right. The scale of the picture may be determined from the approximate tube diameter of 0.9 -in.

Three pinhole punctures caused by the electrical discharge may be noted in figure 6 . One is on the top of the pipe near the TFE insulator bushing. The second is the bright spot in the center of the pipe; and, the third is a bright spot at the bottom of the pipe towards the entrance end. None of these punctures were present prior to the initial breakdown.

When the TFE pipe was cut open to display the inner surface after the initial breakdown, the paths over which the numerous spark discharges occurred were observed. Rather wide and deep transverse and sometimes lateral paths were found to be centered about each pinhole puncture. Lighter paths branched off from these to cover practically the whole interior surface of the pipe with a rectangular network pattern in which the distance between paths was of the order of a few millimeters. The impression gained from this network is that of electrical charges draining along tributary paths to a central stream and thence to a puncture point. Also, it is believed that the paths traced by the spark discharges are proof that discharges such as are shown in figure 6 occur along the inner surface of the pipe and not in the liquid itself.

As flow continued after the initial breakdown, a glow discharge and sometimes small sparks were noted within the pipe centered about one or more of the punctured points. Electricity in the form of a purple corona discharge was seen streaming into the punctured points from the air. This corona discharge continued so long as the fuel flowed and its effect was to enlarge or erode the pinhole punctures.

Although not known definitely, it seems reasonable that the potentials existing just prior to breakdown may have been in the range 50 to $100 \mathrm{kV}$ as this would likely be sufficient to cause the dielectric failure of the pipe wall. The electrical breakdown of nonconductive TFE pipe as described above is repeatable. It was observed in the same manner with numerous pipe samples, and other photographs of these initial breakdown flashes have been obtained.

\subsection{Current-Voltage-Resistance Relationship}

Three samples of TFE pipe with carbon inner liners, designated as $\mathrm{A}, \mathrm{B}$, and $\mathrm{C}$, each having a length of 36 in., were selected for the investigation of the current, voltage, resistance relationship as expressed in eq (3). The end-to-end resistances $R_{T}$ of samples $\mathrm{A}$ and $\mathrm{B}$ are given in figure 3 . The resistances of samples $\mathrm{A}, \mathrm{B}$, and $\mathrm{C}$ were $14,000,600$, and $12 \mathrm{M} \Omega$, respectively, with a test current of $1 \mu \mathrm{A}$. Using the flow circuit of figure 2 , pipe current, $I_{p}$, filter current, $I_{f}$, and pipe potential, $V_{\max }$, were measured. Pintype probes described previously were placed on the test samples at distances of 9,18 , and 27 in. from the upstream end for the measurement of $V_{\max }$. The potential between each of these three probes and the ground was measured with high impedance voltmeters for each flow condition established.

The hydrocarbon naptha had a conductivity of 84 $\mathrm{pmho} / \mathrm{m}$ throughout these tests. Flow rates in the range 5 to $26 \mathrm{gpm}$ were selected. These rates, in combination with the particular fuel conductivity and the inside pipe diameter of 0.875 -in., resulted in values of $\lambda$ in the range 1 to 6 . Pipe currents, $I_{p}$, ranging from $2 \times 10^{-10}$ to $3 \times 10^{-6}$ A could be obtained conveniently by conducting tests with the filter by-pass valve both open and closed over the range of flowrates selected.

The results of these tests are summarized in figure 7 , a log-log plot of the maximum pipe potential, $V_{\max }$, determined from measurements at the three probes, as a function of the current, $I_{p}$, from the pipe to the ground. All of the pipe currents in figure 7 having a magnitude less than $10^{-7} \mathrm{~A}$ were negative. These were obtained with the filter by-pass valve open, a condition of minimum filter charging. It is believed these smaller currents all resulted from the separation of charges at the liquid-pipe interface. The pipe potentials corresponding to these small negative currents were also negative with the single exception of sample $A$ at $5 \times 10^{-10}$ A where positive readings of voltage were obtained. No explanation can be suggested for this single deviation, but it is of interest to note this test point does not lie on the straight line defined by the other points.

All $I_{p}$ currents greater than $10^{-7} \mathrm{~A}$ were positive and were obtained with flow through the filter, i.e., the by-pass valve completely closed. These larger cur- 


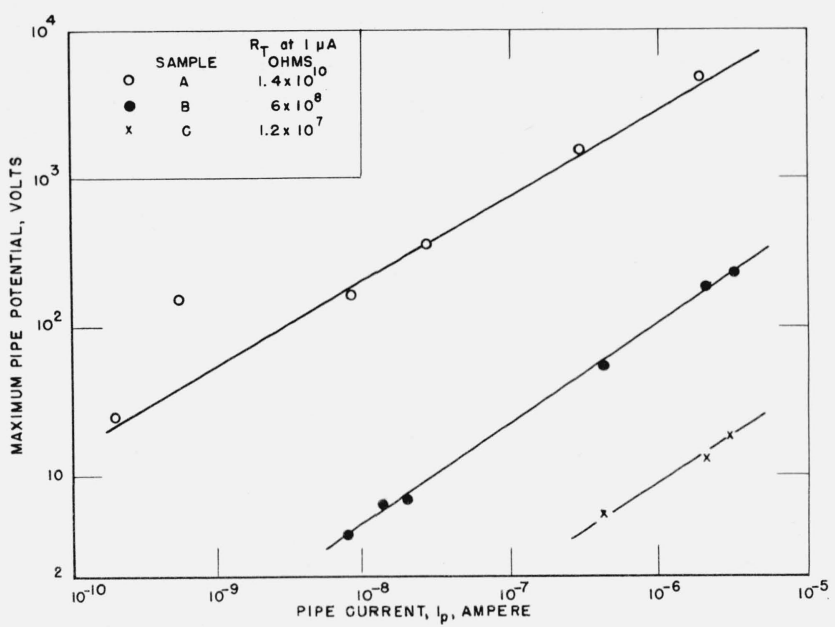

FIGURE 7. Maximum potential versus pipe current in pipes with carbon inner liners (length $36 \mathrm{in}$., flowrate range 5 to $26 \mathrm{gpm}$, fuel conductivity $84 \mathrm{pmho} / \mathrm{m}$ ).

rents were the result of the separation from the fuel to the wall of the pipe of charges produced during flow through the filter. Also, all potentials resulting from the positive currents were likewise positive.

Still referring to figure 7 , it is seen that one straight line for each pipe sample defines the test points quite closely and the slopes of these three lines are approximately equal. Also, the slopes are considerably less than $45^{\circ}$, indicating that the resistances of the three pipe samples were not constant, but tended to decrease with increasing voltage.

The experimentally determined maximum pipe potentials of figure 7 can be compared to those predicted by eq (3) provided $R_{T}$ values corresponding to the pipe currents under consideration are used. Figure 3 gives these values for samples A and B. In selecting a value of $R_{T}$ from this figure which corresponds to a designated $I_{p}$ current, it is assumed that the net deposition of charges is relatively uniform lengthwise along the pipe. Thus, the average current existing in the wall of the pipe can be considered to be approximately $0.25 I_{p}$. This is the current which has been used in obtaining values of $R_{T}$ from figure 3 .

The comparison of calculated and measured maximum pipe potentials for samples $\mathrm{A}$ and $\mathrm{B}$ is presented in table 1. Considering the practical difficulties

TABLE 1. Comparison of calculated and measured maximum pipe voltages

\begin{tabular}{|c|c|c|c|c|}
\hline \multirow{2}{*}{ Sample } & \multirow{2}{*}{$I_{p}$} & \multirow{2}{*}{$\begin{array}{c}R_{T} \\
\text { (fig. } 3 \text { ) }\end{array}$} & \multicolumn{2}{|c|}{ Maximum pipe voltage } \\
\hline & & & $\begin{array}{c}\text { Calculated } \\
V_{\max }=0.1 R_{T} I_{p}\end{array}$ & $\begin{array}{l}\text { Measured } \\
\text { (fig. 7) }\end{array}$ \\
\hline A & $\begin{array}{c}\text { Amperes } \\
10^{-7} \\
10^{-6} \\
10^{-5}\end{array}$ & $\begin{array}{c}\text { Ohms } \\
4 \times 10^{10} \\
2.2 \times 10^{10} \\
1.2 \times 10^{10}\end{array}$ & $\begin{array}{r}\text { Volts } \\
400 \\
2,200 \\
12.000\end{array}$ & $\begin{array}{r}\text { Volts } \\
700 \\
2,700 \\
11,000\end{array}$ \\
\hline B & $\begin{array}{l}10^{-6} \\
10^{-5}\end{array}$ & $\begin{array}{l}9.3 \times 10^{8} \\
4.7 \times 10^{8}\end{array}$ & $\begin{array}{r}90 \\
470\end{array}$ & $\begin{array}{l}100 \\
490\end{array}$ \\
\hline
\end{tabular}

involved in measuring $R_{T}$ and $V_{\max }$ accurately it is believed that good agreement between the calculated and measured values was obtained. Certainly agreement such as this is quite adequate for the practical purpose of estimating potentials to be encountered in pipes through which a known current $I_{p}$ to the ground is encountered. However, it must be realized that eq (3) applies only to the conditions in which the conductive portion of the pipe is in actual contact with the fuel throughout its length and the pipe is in electrical contact with the ground only at the two ends. Grounding of the conductive portion of the pipe at many locations throughout the length will of course result in lower voltages. Insulating the conductive portion of the pipe from the fuel will likely result in potentials having magnitudes sufficient to cause electrical breakdown.

\subsection{Charge Relaxation in Short Pipes}

Both filter current, $I_{f}$, and pipe current, $I_{p}$, were measured during the experiments reported in figures 4 and 7. During that portion of these tests in which the fuel passed through the filter, the values of $I_{f}$ always exceeded $0.5 \mu \mathrm{A}$. These are very large compared to the currents generated at the fuel-pipe interface when the fuel bypassed the filter. Thus, for flow through the filter, it can be assumed that the measured $I_{p}$ values are equivalent to the charges which migrated to the pipe wall during passage of the charged fuel through the pipe. Thus, data are available to verify eq $(2), I_{p} \approx I_{f}\left(1-e^{-\lambda}\right)$.

The results of this check of the relation $I_{p} \approx I_{f}\left(1-e^{-\lambda}\right)$ are shown in figure 8 . Each plotted point is the ratio

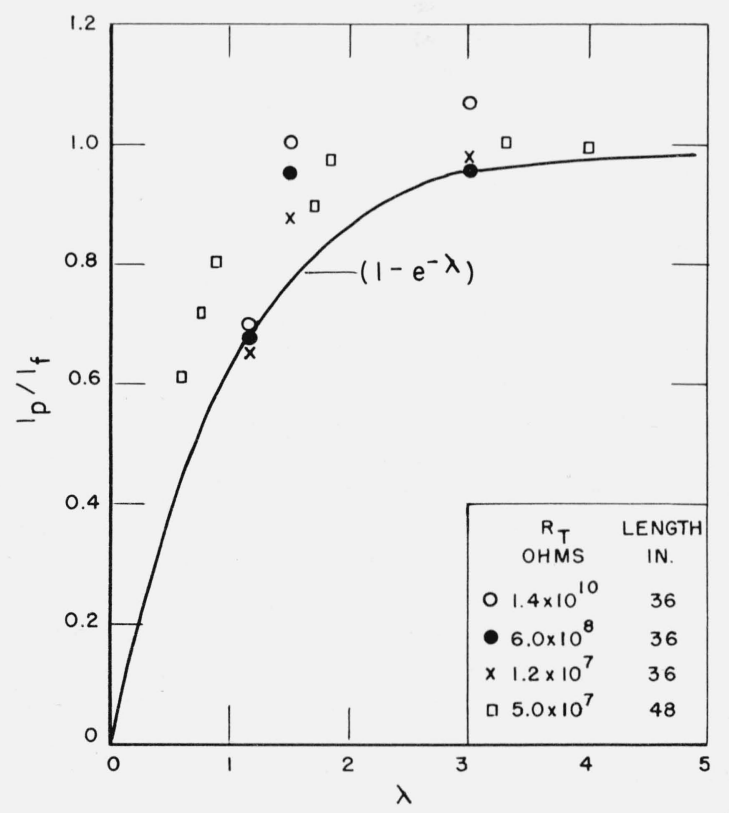

FIGURE 8. Experimental verification of the relation $\mathrm{I}_{\mathrm{p}} / \mathrm{I}_{\mathrm{f}}=\left(1-\mathrm{e}^{-\lambda}\right)$ for various pipe samples, flow rates, and fuel conductivities. 
$I_{p} / I_{f}$ as calculated from the measured values of these two currents. The solid line represents the function $\left(1-e^{-\lambda}\right.$ ) versus $\lambda$, computed from the relation $\lambda=L / v \tau$. Data corresponding to the three pipe samples 36-in. long were all obtained with a constant fuel conductivity of $84 \mathrm{pmho} / \mathrm{m}$, and flowrate in the range 5 to $26 \mathrm{gpm}$. Data for the one pipe sample 48-in. long were obtained at a constant flow of $8 \mathrm{gpm}$ with fuel conductivities in the range 11 to $200 \mathrm{pmho} / \mathrm{m}$ using asphaltenes as the contaminant. The few experimental points obtained for the $\lambda$ range 5 to 10 are not included in this figure because these measured values of $I_{p} / I_{f}$ were all within a few percent of unity. It is believed that the agreement shown in figure 8 is reasonable over the range of conditions encountered. However, some of the points indicate relaxation may at times occur more rapidly than predicted. Certainly these results help to verify that relaxation of a charged liquid flowing in a pipe is essentially independent of the electrical resistance of the pipe for resistance values in the range zero to about $10^{10} \Omega$.

It is believed, but not definitely known, that at least some of the deviations shown in figure 8 are related to the determination of fuel conductivity. Perhaps the effective conductivity of the charged fuel in the pipe can be influenced by the potential gradients existing. Thus, it is possible that present methods of determining fuel conductivity are not completely adequate for the experimental verification of the theoretical relations.

\section{Discussion}

The purpose of this section is to discuss and further emphasize those results leading to the desirable resistance characterisitcs of pipes and hoses through which charged liquid petroleum products may flow. It is believed that the experimental conditions of flowrate, and of pipe diameter, length, and installation downstream from microfilters duplicate closely conditions frequently encountered, especially in fuel systems of the larger size internal combustion engines. Admittedly, this investigation was concerned primarily with the more severe conditions rather than with average conditions. Also, the fuel conductivities of 84 and $165 \mathrm{pmho} / \mathrm{m}$ used during many of the tests are higher than those normally encountered. However, experiments with fuels of lower conductivities show that maximum filter charging effects usually occur in the conductivity range 10 to $200 \mathrm{pmho} / \mathrm{m}$ depending upon the filter design and the rate of flow. Experience has shown that conductivities in the range 10 to $30 \mathrm{pmho} / \mathrm{m}$ are encountered rather frequently in liquid petroleum fuels flowing into the engines.

Perhaps one point should be emphasized concerning fuel conductivity. In investigations concerned with the more severe filter charging conditions and the resultant currents and potentials in pipes installed downstream, it has been found, as shown in figure 4, that greatest electrification effects are obtained with fuels of moderate conductivities, range 10 to 200 $\mathrm{pmho} / \mathrm{m}$, rather than those having low conductivities of the order of one pmho/m. Therefore, elaborate precautions to remove absorbed water and as much of the contamination as possible are not necessary during investigations involving the generation of large filter charging currents. Nevertheless, in this connection some effects of water should be mentioned. As discussed in [3] and investigated briefly by the authors, ordinary tap water, which has relatively very high conductivity, does not increase fuel conductivity even when present to the extent of 5 percent or so. In fact, water frequently tends to leach out and reduce the amount of contaminant in a fuel with the resultant effect of a possibly large decrease in conductivity. Finally, water mixed with fuel, especially when dispersed in the form of droplets, usually increases electrification considerably during flow through pipes and filters.

It has been shown that electrical breakdown can occur when a charged fuel flows through nonconductive pipes. These phenomena are perhaps similar to the charging of an electrical condenser until the resulting potential gradients are sufficient to cause breakdown. Thereafter, depending upon the current and the particular materials involved, either a continuous discharge will be obtained through the breakdown path, or a series of intermittent discharges will result.

The question arises as to what happens when the nonconductive pipe has a sufficiently high dielectric strength so failure through the wall does not occur at the time of the arc discharge. An example of such conditions is described below.

Variable-area meters are used frequently in the laboratory for the flowrate measurement of hydrocarbon liquids. The metering tubes of these instruments are moulded of thick heavy glass, have a length in the range 1 to $3 \mathrm{ft}$, and frequently have no internal bonding wire or its equivalent. Occasionally such meters are connected downstream from microfilters which charge the fuel significantly under certain operating conditions.

It is rather well known that electrical discharges occur at times in glass-tube flowmeters so installed. Photographs of such discharges are very similar in appearance to figure 6 except that puncture or breakage of the glass pipe does not occur. Rather, the strong lengthwise spark terminates at any convenient metallic ground within the end of the glass tube. Numerous lateral flashes are noted also. Although not definitely known, it appears from photographs, that the electric arcs occur on the internal surfaces of the glass pipe. Arcs extending five or more inches up into the glass tubes have been observed under the more severe conditions.

The use of an internal bonding or ground wire in nonconducting pipes should reduce maximum potentials significantly and thus eliminate the possible hazard arising from electrical arc discharges. This internal bonding wire will likely have little, if any, influence upon the rate at which separation of charge from the highly charged fuel occurs.

The possibility of placing a conductive skin around the outside of nonconductive hoses was mentioned 
in the Discussion of reference [2]. This idea may be practical provided the transverse leakage of electricity from the interior of the hose to the conducting skin does not puncture the wall. Experience has demonstrated this is not practical when applied to hoses containing a thin tube of pure TFE surrounded by an exterior, grounded, metal braid. In such hoses, discharges of electricity similar to that of figure 6, frequently puncture the TFE tube resulting in liquid leakage through the wall. One solution to the problem is the development of a practical inner liner or coating of moderate resistivity which would be grounded at each end of the hose.

Considering now the flow of a highly charged liquid hydrocarbon through a pipe of moderate resistivity, the experimental results help to verify that the rate of charge separation from the fuel to the pipe wall is essentially independent of the pipe material or of its electrical resistance. The relation $I_{p}=I_{i}\left(1-e^{-\lambda}\right)$ is in reasonable agreement with the experimental results provided $I_{i}$ is large compared to $I_{0}$, where $I_{0}$ is the convection current flowing out with the fuel from an infinitely long pipe of the same material.

The maximum magnitudes of the currents $I_{p}$ encountered in the investigation were in the range 1 to $6 \mu \mathrm{A}$. These are of the same order of magnitude and are in reasonable agreement with a possible maximum value of $10 \mu \mathrm{A}$ cited at times in the literature. If a safety factor is desired, a maximum value of $I_{p}=100$ $\mu \mathrm{A}$ will perhaps cover all operating conditions which could be encountered in practical applications.

The rather limited experiments indicate that the maximum voltages occurring in pipes can be predicted with reasonable accuracy by the relation $V_{\max }=0.1 R_{T} I_{p}$ provided $\lambda<6$. According to eq (5) in the appendix and as shown in figure 9 , for any given value of $R_{T}$ and $I_{p}$, maximum potentials will be less for values of $\lambda$ considerably greater than 6 . However, such conditions are not usually encountered in practical applications. If potentials existing within pipes are not to exceed an arbitrary value of $1,000 \mathrm{~V}$ for a possible maximum pipe current of $100 \mu \mathrm{A}$, then the end-to-end resistance of the pipe may be computed as $R_{T}=1,000 /$ $\left(0.1 \times 100 \times 10^{-6}\right)=10^{8} \Omega$.

Thus, a value of $R_{T}$ not to exceed about $10^{8} \Omega$ may be suggested for pipes and hoses intended for use with liquid hydrocarbons. It is believed this upper limit is sufficiently low to eliminate the hazard of spark discharges occurring either within or through the walls of hoses and pipes through which a liquid hydrocarbon flows. However, this suggested maximum value for $R_{T}$ is subject to three conditions: (a) the pipe must be uniformly conductive throughout its length; (b) the conductive portion of the pipe must be in contact with the fuel throughout the length of the pipe; and (c) the conductive portion of the pipe must be electrically connected to the ground at each end of the pipe.

When conventional end fittings of aircraft hose are used as electrodes, the end-to-end resistance $R_{T}$ is not a reliable parameter for specification purposes because it does not assure that the interior surface of the pipe is conductive and electrically connected to the ground. However, this requirement will be checked if the resistance from each and any portion of the interior surface of the pipe to the ground is measured instead of the end-to-end resistance. Consider an empty pipe grounded at each end. An internal electrode located at the center of this pipe has the effect of placing two $R_{T} / 2$ resistances in parallel resulting in an effective resistance to the ground of $R_{T} / 4$. With allowance for this, and considering orders of magnitude only, it is concluded that hazardous pipe potentials resulting from static electricity can be eliminated if the electrical resistance from each and any portion of the interior surface of the pipe to the ground does not exceed about $10^{7} \Omega$. The use of an internal electrode in measuring the resistance of pipes and hoses will be inconvenient at times, but this method does provide an electrical path to the ground similar to that seen by the liquids contained within the pipe during normal usage.

It is acknowledged without reservation that the relations and ideas presented in [1] and [2] and their Discussions provided nearly all of the background material required for this investigation. It merely remained necessary to demonstrate that such relations and ideas were applicable to the particular problem under consideration. Thus, it is gratifying to be able to present additional experimental data which, for practical purposes, is in good agreement with the work of previous investigators.

\section{Conclusions}

1. High electrical charge densities can be produced in petroleum liquids having moderate to high electrical conductivities (10 or more $\mathrm{pmho} / \mathrm{m}$ ) when such liquids flow through conventional microfilters. However, the charging tendency of the liquid is not dependent upon fuel conductivity alone. Rather. it is dependent upon the amount and material of which the contaminant is composed and the material at the liquid-solid interface.

2. Maximum currents in the range one to $6 \mu \mathrm{A}$ were obtained from the pipe to the ground when highly charged petroleum liquids flowed through short pipes or hoses. These comparatively large currents were caused by the migration to the wall of the pipe of the electrical charges contained within the charged fuel.

3 . The rate of separation of electrical charges from the charged fuel is essentially independent of the pipe material and its electrical resistance.

4. When the rate of charge separation is sufficiently high in pipes constructed from nonconductive materials, electrical discharges in the form of sparks and arcs will occur. These discharges will originate on the inner surface of the pipe. They may be contained entirely within the pipe or may cause puncture or other failure of the pipe wall depending upon the pipe material, the wall thickness, and the ground locations involved.

5. Maximum potentials produced by static electric phenomena within pipes, grounded at each end, may be predicted with reasonable accuracy by a simple 
relation provided the electrical resistance of the pipe and its current to the ground are known.

6. Considering pipes or hoses through which a highly charged petroleum liquid may flow, an electrical resistance from each and any portion of the interior surface of the pipe to the ground not exceeding about $10^{7} \Omega$ is adequate to limit pipe potentials resulting from static electricity to less than $1,000 \mathrm{~V}$.

The financial support and personal interest shown during this program by the Navy Department, Bureau of Naval Weapons, is gratefully acknowledged. Also, the appreciation of the authors is hereby expressed for the considerable technical guidance received throughout the program from Francis Hermach of the Electricity Division of the National Bureau of Standards. Finally, the considerable contribution of industry in supplying experimental pipe samples for the investigation is also acknowledged.

\section{References}

[1] Carruthers, J. A. and Marsh, K. J., Charge relaxation in hydrocarbon liquids flowing through conducting and nonconducting pipes, J. Institute of Petroleum 48, No. 462, June 1962, pp. 169-179.

[2] Carruthers, J. A. and Wigley, K. J., The estimation of electrostatic potentials, fields, and energies in a rectangular metal tank containing charged fuel, J. Institute of Petroleum 48, No. 462, June 1962, pp. 180-195.

[3] Klinkenberg, A. and van der Minne, J. L. (ed), Electrostatics in the petroleum industry, Amsterdam: Elsevier, 1958.

\section{Appendix}

The object of this appendix is to develop an approximate relation for the maximum electrical potentials developed on the inner wall surface of pipes as a result of static electric phenomena caused by the flow of hydrocarbon liquids. Essentially this theory is a simplification of that developed by Carruthers and Marsh [1] subject to the assumptions that the electrical resistance of the pipe is low compared to that of the liquid and that the ratio of the residence time of the liquid in the pipe to the relaxation time of the liquid has a value less than 6 . Both of these assumptions appear to be valid as applied to the more severe conditions likely to be encountered in practical applications.

$I_{f}=$ current between the ground and the filter

$I_{i}=q_{i} v=$ convection current entering the pipe due to the flow of liquid containing an initial charge $q_{i}$ per unit length of pipe

$I_{p}=$ current flowing from the pipe to the ground

$I_{0}=$ convection current which would emerge with the liquid from an infinitely long pipe

$L=$ length of pipe

$m=x / L$

$R_{T}=L / \beta=$ end-to-end resistance of the pipe

$V_{m}=$ the potential on the inner wall of the pipe at a position $m=x / L$ from the pipe entrance
$V_{\max }=$ the maximum potential existing on the inner wall of the pipe

$V_{x}=$ the potential on the inner wall of the pipe at a distance $x$ from the pipe entrance

$v=$ mean axial velocity of the liquid

$x=$ distance along pipe from the entrance

$\beta=$ conductance per unit length of pipe wall assuming the conductivity of the pipe wall is considerably greater than that of the liquid

$\lambda=L / v \tau=$ ratio of the residence time for the liquid in the pipe to the relaxation time of the liquid

$\tau=$ relaxation time of the liquid. Its value is computed from $e e_{0} / k$ where $k$ is liquid conductivity, $e$ the dielectric constant of the liquid, and $e_{0}$ the electric constant of free space. When $k$ is expressed in $\mathrm{mho} / \mathrm{m}, \tau$ in seconds, and $e$ is taken as $2, \tau=17.7 \times 10^{-12} / \mathrm{k}$.

Carruthers and Marsh [1] in a consideration of the potentials produced by the flow of hydrocarbon liquids have developed the expression

$$
V_{x}=\left(I_{i}-I_{0}\right) \frac{v \tau}{\beta}\left[\left(1-e^{-x / v \tau}\right)-\frac{x}{L}\left(1-e^{-L / v \tau}\right)\right]
$$

for the potential at any point of the cross section of liquid and pipe.

If it is assumed that the electrical resistance of the pipe is small compared to that of the liquid contained within the pipe, $R_{T}=L / \beta$, and by letting $m=x / L$ and $\lambda=L / v \tau$, eq (1) may be written as

$$
V_{m}=\left(I_{i}-I_{0}\right) \frac{R_{T}}{\lambda}\left[\left(1-e^{-m \lambda}\right)-m\left(1-e^{-\lambda}\right)\right] .
$$

Also in [1], the expression

$$
I_{u}=I_{i} e^{-\lambda}+I_{0}\left(1-e^{-\lambda}\right)
$$

is developed for the convection current, $I_{u}$, emerging with the liquid when the pipe is of finite length. This assumes both the relaxation of $I_{i}$ and the generation of $I_{0}$ at the fuel-pipe interface are exponential processes. As shown in the discussion of figure $1, I_{u}=I_{i}-I_{p}$, and from eq (3) the currents $I_{p}, I_{i}$, and $I_{0}$ are related by the expression

$$
I_{p}=\left(I_{i}-I_{0}\right)\left(1-e^{-\lambda}\right) .
$$

Substituting for $\left(I_{i}-I_{0}\right)$ in eq (2) another expression

$$
V_{m}=I_{p} \frac{R_{T}}{\lambda}\left[\frac{\left(1-e^{-m \lambda}\right)}{\left(1-e^{-\lambda}\right)}-m\right]=K I_{p} R_{T}
$$

is obtained for the potential at any point along the pipe.

Figure 9 shows $K$ plotted as a function of $\lambda$ for selected values of $m$. It is seen that $K$ varies between 0.13 and 0.08 for values of $\lambda$ less than 6 . As $\lambda$ becomes greater than $6, K$ decreases rapidly becoming less 


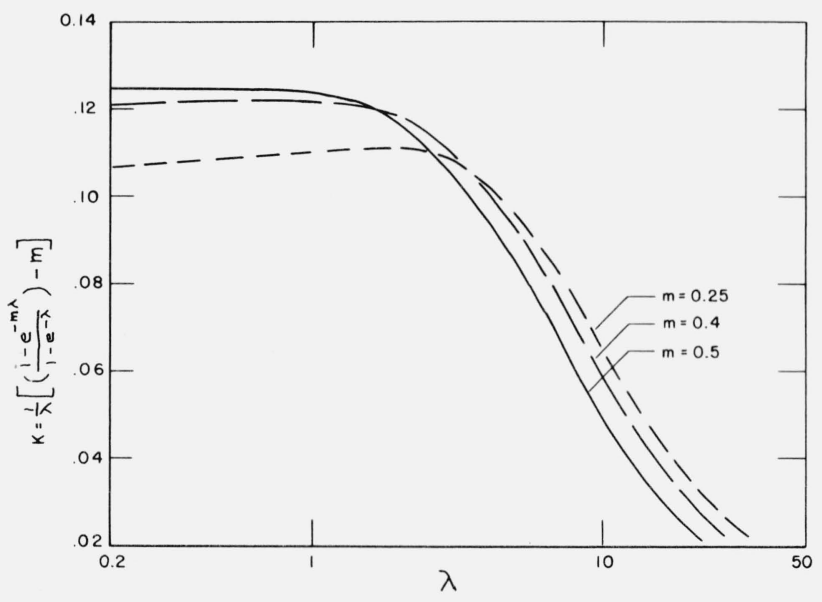

FigURE 9. The relation $\mathrm{K}=\frac{1}{\lambda}\left[\frac{\left(1-\mathrm{e}^{-\mathrm{m} \lambda}\right)}{\left(1-\mathrm{e}^{-\lambda}\right)}-\mathrm{m}\right]$ as a function of $\lambda$ for selected values of $\mathrm{m}$. than 0.02 for $\lambda=50$. Values of $\lambda$ exceeding 6 are encountered only with long pipes, fuels of very high conductivities, or very low fuel velocities; conditions in which nearly all of the charge relaxation occurs in a short upstream portion of the pipe. Values of $\lambda$ less than 6 correspond to the more severe conditions encountered in practical applications. Therefore, with little sacrifice in accuracy an average value of $K=0.1$ can be used to obtain an approximate expression for the maximum potential produced in a pipe, and

$$
V_{\text {max }}=0.1 R_{T} I_{p}
$$

where $R_{T}$ is the end-to-end resistance of a pipe, grounded at each end, from which a current $I_{p}$ is flowing to the ground.

(Paper 69C4-212) 